\title{
一个 $Z_{p}$-Borsuk-Ulam 定理
}

\author{
王志 强 \\ (北京大学数学系)
}

\section{关请词等变映射、 $Z$ ，作用、Borsuk-Ulam 定理}

本文讨论了关于 $Z$, 作用等变映射拓扑度的计算, 并给出 $Z$, 作用的 Borsuk-Ulam 定 理.

首先给出一些符号. 对非负整数 $m, n,\langle m, n\rangle$ 表示 $m$ 和 $n$ 的最大公约数. $m \mid n$ 表示 $m$ 是 $n$ 的因子. 以下固定正整数 $p$, 并设

$$
p=p_{1}^{r} \cdots p_{s}^{r} \text {. }
$$

这里 $p_{1}<p_{1}<\cdots<p_{s}$ 是 $p$ 的素因子, $r_{i}>0$ 整数, $i=1,2, \cdots, s$.

先来考虑 $Z_{p}$-Borsuk 定理. 在 $\mathrm{C}^{n}$ 上给出两个 $Z_{p}$ 作用如下:

$$
\begin{aligned}
& z \mapsto T z=\left(e^{i m_{1} 2 \pi / p} z_{1}, \cdots, e^{i m_{n} 2 \pi / p_{n_{n}}}\right), T^{p}=\mathrm{Id}, \\
& z \mapsto \widetilde{T} z=\left(e^{i k_{1} 2 \pi / p} z_{1}, \cdots, e^{i k_{n} 2 \pi / p} z_{n}\right), \widetilde{T}^{p}-\mathrm{Id},
\end{aligned}
$$

这里 $m_{j} \neq 0, k_{i} \neq 0$ 都是整数, $j=1,2, \cdots, n$.

设 $Q \subset \mathrm{C}^{n}$ 是原点 $\theta$ 的一有界开邻域, 且是关于作用 $T$ 不变的. 设 $f: Q \rightarrow \mathrm{C}^{n}$ 是一连续 映射, 如果对任何 $z \in Q, f(T z)=\widetilde{T} f(z)$, 则称 $f$ 是关于 $(T, \widetilde{T})$ 的等变映射. 或者用分量 表示

$$
f_{j}(T z)=e^{i k_{i} / \alpha / p} f_{i}(z), i-1,2, \cdots, n \text {. }
$$

记

$K-\left|k_{1}\right|, \cdots,\left|k_{n}\right|$ 的最小公倍数，

$M-\left|m_{1}\right|, \cdots,\left|m_{n}\right|$ 的最大公约数,

$\gamma_{M K}-p /\langle p, M, K\rangle,\langle p, M, K\rangle=p, M, K$ 的最大公约数.

定现 1 按以上符号和假设, 若 I: $Q \rightarrow C^{n}$ 是关于 $(T, \widetilde{T})$ 的等变映射, $\forall A f(\partial 0)$, 则

$$
\operatorname{deg}(f, \Omega, \theta)=\prod_{i=1}^{n} \frac{k_{i}}{m_{i}}\left(1+a \cdot \gamma_{M K} \cdot(\langle M, K\rangle)^{n}\right),
$$

这里 $\propto$ 是依赖于 $f$ 的一个整数.

注 1. 关于等变映射拓扑度的计算已有很多工作，但几乎所有结果都是讨论两边空间上 的作用一样的情形(即 $T=\widetilde{T}$ ), 在文献 $[1,2]$ 中我们给出了在任意紧 Lie 群作用下这种情况 下的结果. 文献 [3]中讨论了 $S^{1}$ 群作用下两边作用不同的情形,对其他群作用尚无结果.

证 证明的主要想法与文献 [3]中是一致的. 不失一般性,不妨设所有 $m_{i}>0, k,>0$, $i-1,2, \cdots, n$. 否则可用变量替换把 $z_{i}$ 换为复共轭 $\bar{z}_{i}$ (或把 $f_{i}$ 换为 $f_{i}$ ). 记 $M_{1}-\langle M, K\rangle$,

本文 1987 年 10 月 2.3 日收到. 


\section{再令}

$$
\begin{aligned}
& g(t)=\left(t_{1}^{m}, / M_{1}, \cdots, t_{n}^{m} n^{\prime M M_{1}}\right), \\
& \tilde{\boldsymbol{g}}(t)=\left(t_{1}^{K / k_{1}}, \cdots, t_{n}^{K / k_{n}}\right) .
\end{aligned}
$$

这里 $\forall z \in Q_{1}, g(z) \in Q$, 显然 $Q_{1}$ 仍为 $\theta$ 的一有界开邻域, 且关于作用 $T-e^{i W_{1}^{2} / / P I d}$ 不 变. 由拓扑度计算的乘积公式 $\left(\Omega_{2}\right.$ 类似定义):

$$
\begin{aligned}
\operatorname{deg}\left(h, \Omega_{1}, \theta\right) & =\operatorname{deg}\left(\tilde{g}, \Omega_{2}, \theta\right) \cdot \operatorname{deg}(f, \Omega, \theta) \cdot \operatorname{deg}\left(\dot{g}, \Omega_{1}, \theta\right) \\
& =\prod_{i=1}^{n} \frac{k_{i}}{k_{i}} \cdot\left(\frac{K}{M_{1}}\right)^{n} \cdot \operatorname{deg}(f, \Omega, \theta) .
\end{aligned}
$$

又直接计算知 $h$ 满足

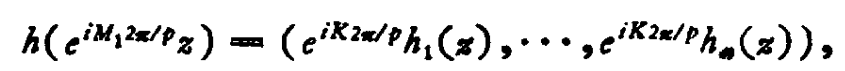

因此只需考虑所有 $m_{i}=M_{1}, k_{i}-K$ 的情形.

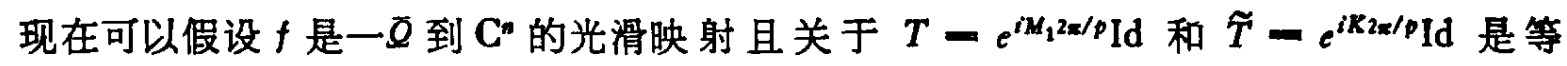
变的. 事实上, 由多项式逼近定理, $\forall \varepsilon>0$ 有光滑映射 $f^{*}$ 满足 $\left\|f-f^{*}\right\|_{c(\theta)}<8$. 再平均 $f^{*}$ :

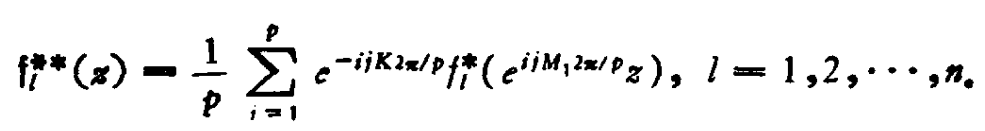

易查 $f^{* *}$ 是一关于 $T$ 和 $\widetilde{T}$ 等变的光滑映射, 并且

$$
\begin{gathered}
\left\|f_{l}^{* *}(\boldsymbol{z})-f_{l}(\boldsymbol{z})\right\| \\
\leqslant\left\|\frac{1}{p} \sum_{i=1}^{p} e^{-i i K_{z} / p / p}\left[f_{l}^{*}\left(e^{i j M_{1} 2 z / p_{z}}\right)-f_{l}\left(e^{i j M_{1} 2 z / p_{z}}\right)\right]\right\| \leqslant 8_{0}
\end{gathered}
$$

现在仍记 f** 为 f 并设其具有 $f^{* *}$ 的性质. 下面取 $\delta>0$ 充分小，定义 $-R_{+}$到 $R_{+}$的非 增光滑函数 $\eta$, 满足当 $t \leqslant \frac{\delta}{2}$ 时, $\eta(t)-1$, 当 $t \geqslant \delta$ 时, $\eta(t)=0$.

令

$$
f(x)-\eta(|z|)\left(z_{1}^{\mathbb{R} / M_{1}}, \cdots, z_{0}^{\mathbb{R} / M_{1}}\right)+(1-\eta(|z|)) f(z),
$$

则易见 7 也是 $\Omega$ 到 $C^{n}$ 的关于 $T$ 和 $\widetilde{T}$ 等变的光滑映射, 且当 8 充分小时

在原点附近 $\tilde{f}(x)-\left(z_{1}^{K / M_{1}}, \cdots, z_{n}^{R / M_{1}}\right)$. 定义

$$
\operatorname{deg}(\tilde{\mathfrak{f}}, \theta, \theta)-\operatorname{deg}(\mathfrak{f}, \varepsilon, \theta) \text {, }
$$

$$
F_{i}(z, A)=t_{i}(x)+\sum_{i=1}^{n} A_{i l} z_{t}^{R / M_{1}}, j-1,2, \cdots, n_{0}
$$

这里 $A \in \mathfrak{M} \triangleq\left\{B \in L\left(\mathbb{C}^{n}\right) \mid\|B\|<\delta / 8\right\}$.

记 $\Xi-\left\{z \in O \mid\|z\|>\frac{\delta}{4}\right\}$, 与文献 [3] 中类似的讨论可以证明 $F: \delta \times \mathfrak{M} \rightarrow \mathrm{C}^{n}$ 是与 $\theta \in \mathrm{C}^{n}$ 横截的, 由横截定理 ${ }^{[4]}$, 存在糔中一稠密集合, 若 $A$ 属于此集合, $\theta$ 是 $F(\cdot, A)$ 的正则 值. 取 $\|A\|$ 充分小,只须计算 $\operatorname{deg}(F(\cdot, A), \Omega, \theta)$ 即可.

注意到 $F(\cdot, A)$ 仍是关于 $T$ 和 $\widetilde{T}$ 等变的咉射, 及 $\theta$ 是 $F$ 的正则值, 故在 $\widetilde{\Omega}$ 内 $F$ 仅有有 限个零点轨道, $N_{1}, \cdots, N_{\mathrm{m}}$. 而在 $Q \backslash \tilde{Q}$ 上,由同伦不变性 


$$
\begin{aligned}
\operatorname{deg}( & F(\cdot, A), \mathcal{Q} \backslash \tilde{\Omega}, \theta)=\operatorname{deg}(F(\cdot, 0), Q \backslash \tilde{Q}, \theta) \\
& =\operatorname{ind}(\tilde{\mathfrak{f}}, \theta)=\left(\frac{K}{M_{1}}\right)^{n} .
\end{aligned}
$$

记 $N_{i}=\left\{T^{l} z_{i} \mid l=1,2, \cdots, p\right\}$, 则易知由 $T$ 和 $\widetilde{T}$ 的具体形式, 每个 $N_{i}$ 恰好含有

$$
\gamma_{M K}=p /\left\langle p, M_{1}\right\rangle=p /\langle p, M, K\rangle
$$

个点, 即 $\left|N_{i}\right|-\gamma_{M K}, i=1,2, \cdots, m$. 现取 $N_{i}$ 的邻域 $U_{i}$, 则

$$
\begin{aligned}
\operatorname{deg} & (F(\cdot, A), \Omega, \theta) \\
& -\left(\frac{K}{M_{1}}\right)^{n}+\sum_{i=1}^{m} \operatorname{deg}\left(F(\cdot, A), U_{i}, \theta\right) .
\end{aligned}
$$

取定一个 $N_{i}$, 设 $N_{i}=\left\{a_{1}, \cdots, a_{r_{M K}}\right\}$, 由 $\theta$ 是 $F(\cdot, A)$ 的正则值及 Leray-Schauder 指标公式

$$
\operatorname{deg}\left(F(\cdot, A), U_{i}, \theta\right)=\sum_{l=1}^{T_{M K}} \operatorname{sgn}\left[\operatorname{det} D_{z} F\left(a_{l}, A\right)\right] .
$$

但由 $F$ 对 $z$ 等变性, 直接计算(见文献 $[1]$ ) 有

$$
D_{z} F\left(e^{i M_{1} 2 \pi / p} \mathrm{Id} z, A\right)=e^{i K 2 \pi / p} \mathrm{Id} \cdot D_{z} F(z, A) \cdot e^{-i M_{1} 2 z / p} \mathrm{Id} \text {. }
$$

若把 $e^{i K 2 m / p} \mathrm{Id}$ (或 $e^{-i M_{1} 2 \approx / p} \mathrm{Id}$ ) 等同于一个实的 $2 n \times 2 n$ 矩阵,可知此矩阵行列式为 1 . 因此

$$
\operatorname{det} D_{s} F\left(a_{l}, A\right)=\operatorname{det} D_{s} F\left(a_{1}, A\right), \forall l=1,2, \cdots, \gamma_{M K}
$$

从而

最后得到

$$
\operatorname{deg}\left(F(\cdot, A), U_{i}, \theta\right)=\gamma_{M K} \operatorname{sgn}\left[\operatorname{det} D_{z} F\left(a_{1}, A\right)\right]
$$

$$
\begin{aligned}
\operatorname{deg}(i, Q, \theta) & =\left(\frac{K}{M_{1}}\right)^{n}+\gamma_{M K} \sum_{i=1}^{m} \operatorname{sgn}\left[\operatorname{det} D_{x} F\left(z_{i}, A\right)\right] \\
& =\left(\frac{K}{M_{1}}\right)^{n}+\alpha \cdot \gamma_{M K} .
\end{aligned}
$$

类似方法还可证明更一般的结果 (允许不动点空间的存在):

设 $Q \subset \mathrm{C}^{n} \times R^{b}$ 是原点的一个有界开邻域, 令

$$
z=\left(z^{\prime}, z^{\prime \prime}\right), z^{\prime}=\left(z_{1}, \cdots, z_{n}\right), z^{\prime \prime}=\left(z_{n+1}, \cdots, z_{n+b}\right),
$$

这里对 $i>n, z_{i}$ 表实数. 考虑如下 $Z$, 作用:

$$
z 1 \longrightarrow T z=\left(e^{i m_{1} 2 \pi / p} z_{1}, \cdots, e^{i m_{n}^{2 \pi / p} z_{n}}, z_{n+1}, \cdots, z_{n+b}\right) \text {, }
$$

这里 $m_{i} \neq 0$ 整数， $j=1,2, \cdots, n$ 。设 $\Omega$ 关于作用 $T$ 不变.

定理 $1^{\prime}$ 设 $t: \theta \rightarrow \mathrm{C}^{n} \times R^{b}$ 满足

$$
\begin{aligned}
& f_{i}(T z)=e^{i k_{i} 2 z / p} f_{i}(z), k_{i} \neq 0 \text { 整数, } j=1,2, \cdots, n ; \\
& f_{i}(T z)=f_{i}(z) \text { 实函数, } i-n+1, \cdots, n+b .
\end{aligned}
$$

再设对任何点 $z=\left(0, z^{\prime \prime}\right) \in \partial Q$

$$
f_{i}(z)=z_{i}, \quad j-n+1, \cdots, n+b
$$

如果 $\theta \in f(\partial \Omega)$, 则 


$$
\operatorname{deg}(f, \rho, \theta)=\prod_{i=1}^{n} \frac{k_{i}}{m_{i}}\left(1+\alpha \cdot \gamma_{M K} \cdot\left(\frac{\langle M, K\rangle}{K}\right)^{n}\right),
$$

其中符号的意义与定理 1 相同。

下面给出一充分条件保证上述拓扑度不为零. 首先把 $K, M$ 分解为

$$
\begin{aligned}
& K=\widetilde{K} \cdot p_{p_{1}}^{t_{1}} \cdots p_{s}^{t_{s}},\langle\widetilde{K}, p\rangle=1, t_{j} \geqslant 0 j-1,2, \cdots, s ; \\
& M=\widetilde{M} p_{1}^{i_{1}} \cdots p_{s}^{s_{s}},\langle\widetilde{M}, p\rangle=1, l_{i} \geqslant 0, j-1,2, \cdots, s_{.}
\end{aligned}
$$

推论 1 在定理 1 (或定理 $1^{\prime}$ ) 中, 设存在一个 $j, 1 \leqslant i \leqslant s$, 使得

$$
n\left[t_{i}-\left(t_{i} \wedge l_{i}\right)\right]<r_{i}-\left(t_{i} \wedge l_{i} \wedge r_{i}\right),
$$

这里 $t_{i} \wedge l_{i} \wedge r_{i}=\min \left\{t_{i}, l_{i}, r_{i}\right\}$, 则

$$
\operatorname{deg}(f, \Omega, \theta) \neq 0 \text {. }
$$

证 由上述条件可推得 $p_{i}$ 是 $\alpha \cdot \gamma_{M K}\left(\frac{\langle M, K\rangle}{K}\right)^{n}$ 的一个因子, 由表达式易见 $\operatorname{deg}(f$, $\boldsymbol{\Omega}, \theta) \neq 0$.

注 2. 应用中经常是某个 $l_{i}=0$ 或 $t_{i}-l_{i}$, 从而条件可大大简化.

下面给出一个 $Z$,-Borsuk-Ulam 定理.

定理 2 设 $T$ 是如文章开始给定的 $\mathrm{C}^{n}$ 上的 $Z$ ，作用， $Q \subset \mathrm{C}^{n}$ 是一原点的有界开不变 邻域。设 $1: 20 \rightarrow C^{m}$ 是一连续映射, 满足

$$
f_{h}(T z)=e^{i k_{h}^{2} z^{\prime} p} f_{h}(z), h=1,2, \cdots, m,
$$

$k_{h} \neq 0$ 整数. 设 $K, M$ 如上符号并分解, 假设存在 $-i, 1 \leqslant i \leqslant s$, 使得

$$
n\left[t_{i}-\left(t_{i} \wedge l_{i}\right)\right]<r_{i}-\left(t_{i} \wedge l_{i} \wedge r_{i}\right),
$$

若 $m<n$, 则 $\theta \in f(\partial Q)$.

证 由推论 1 和经典 Borsuk-Ulam 定理的证明方法立得.

注 3. 类似于定理 $1^{\prime}$ ，也可给出带不动点空间的 Borsuk-Ulam 定理,留给读者.

[1] Wang, Z. Q., Symmetries and the calculations of degree, Research Memorandum,lnst. of Math. Acade mis Sinica., 1986, No. 44.

[2]王志强,对称生与非线性分析中的一些问题,博士论文, 1986 .

[3] Nirenberg, L., A comments on nonlinear problems, 1981 (preprint).

[4] Guillemin, V. \& Pollack, A., Differential Topology, 1974. 